\title{
Morphogènes et endocytose
}

> Les protéines signalisatrices des familles Wingless/Wnt, Hedgehog et Decapentaplegic (Dpp)/Bone morphogenic protein (BMP) agissent comme des morphogènes au cours du développement. On sait aujourd'hui que ces molécules sécrétées agissent directement sur des cellules pourtant distantes de leurs sites de production, mais peu de choses sont connues sur la façon dont ces molécules se propagent au sein d'un tissu, et sur les mécanismes contrôlant cette propagation. Des travaux récents réalisés chez la drosophile, mêlant des approches génétiques à des approches de biologie cellulaire, ont permis de révéler que le processus d'endocytose joue un rôle central dans le contrôle de la propagation d'un morphogène. <

$(\rightarrow) \mathrm{m} / \mathrm{s}$ $1999, n^{\circ} 11$, p. 1279
Au cours du développement, chaque cellule au sein d'un tissu sait où elle se trouve, et adopte un destin approprié à la position qu'elle occupe: on dit qu'elle possède une information de position. Généralement, cette information de position est fournie par un groupe de cellules particulières, l'organisateur, sous la forme d'un signal morphogène formant un gradient de concentration au sein du tissu. Ainsi, les cellules «mesurent » la distance qui les sépare de l'organisateur grâce à la concentration locale du signal morphogène à laquelle elles sont soumises [1]. Dès le début du siècle dernier, plusieurs embryologistes ont suspecté l'existence de ces gradients. Mais ce n'est qu'au cours de ces quinze dernières années que quelques molécules ont pu être qualifiées de morphogènes.

À l'heure actuelle, une molécule sécrétée est qualifiée de morphogène si elle agit directement sur des cellules distantes de sa source et si elle induit, au niveau de ces cellules, des réponses distinctes selon sa concentration. Chez la drosophile, les protéines sécrétées Wingless, Hedgehog et Decapentaplegic (Dpp) (membre de la famille des TGF- $\beta$ ) remplissent ces conditions. Par exemple, les disques imaginaux de la larve, qui formeront les appendices de l'adulte, sont constitués d'un compartiment antérieur et d'un compartiment posté-

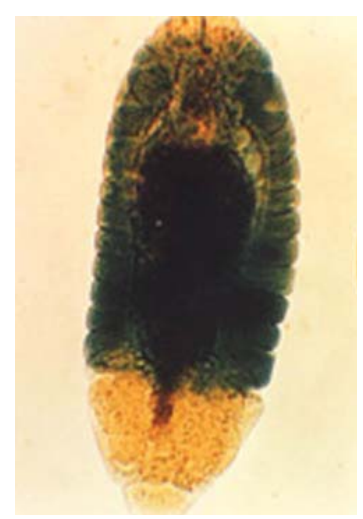

rieur. Dpp est exprimé dans le compartiment antérieur au sein d'une fine bande de cellules située le long de la frontière antéro-postérieure (Figure 1). Dpp est absolument requis pour l'organisation des disques et le développement des appendices $(\rightarrow)$. Dans les cellules voisines de son lieu de production, Dpp active l'expression des gènes spalt et optomotorblind (omb). Mais la concentration de Dpp requise pour activer spalt est plus forte que celle requise pour activer optomotorblind. II en résulte que spalt est exprimé dans les cellules situées près de la source de Dpp, alors que optomotorblind est exprimé dans un domaine cellulaire plus large, également centré sur les cellules exprimant Dpp (Figure 1). La présence du récepteur de Dpp Thikvein (Tkv) est nécessaire pour activer spalt et optomotorblind dans tous leurs domaines d'expression. Ce résultat indique que l'activation de ces gènes dépend de la réception du signal Dpp, y compris dans les cellules les plus distantes de son lieu de production, suggérant que Dpp doit atteindre directement ces cellules. C'est sur la base de ces observations que Dpp a été qualifié de morphogène [2, 3]. Cependant, même si Dpp se définit fonctionnellement comme un 
morphogène, il s'est avéré extrêmement difficile d'observer directement, par des techniques immuno-cytochimiques, le gradient qu'il est censé former. Cette remarque vaut également pour Wingless, Hedgehog et la plupart des morphogènes. Or, si l'on ne peut observer ces gradients, il devient difficile d'étudier les mécanismes qui contrôlent leur formation et leur propagation dans un tissu.

\section{Mécanismes contrôlant la « mise en forme 》 du gradient}

Schématiquement, la forme d'un gradient de concentration d'un morphogène dans un tissu dépend du taux de sécrétion du morphogène (aspect non abordé ici), de la vitesse à laquelle il se propage dans le tissu et de sa stabilité.

La vitesse de propagation d'un morphogène est fonction de la façon dont il est transporté dans le tissu. Le mécanisme de propagation le plus simple est la diffusion passive. Cependant, dans de nombreux cas, il est peu probable qu'un morphogène diffuse librement dans l'espace extracellulaire d'un épithélium. En effet, les gradients se forment parfois dans le plan d'épithéliums très « repliés », comme dans le cas des disques imaginaux de la drosophile. De plus, des molécules sécrétées comme Wingless « collent » aux membranes cellulaires, via l'association à des composants de la matrice extracellulaire et/ou à leurs récepteurs $[4,5]$. Plusieurs mécanismes de transport autres que la libre diffusion ont donc été proposés, tels que la diffusion facilitée dans l'espace extracellulaire, grâce à la présence de molécules «porteuses» comme les protéoglycanes [6]. D’autres encore ont proposé un mécanisme de transport actif similaire à la transcytose, dans lequel le ligand serait transporté d'un endroit à un autre de la membrane de la cellule, au sein des vésicules par des cycles successifs d'endocytose et d'exocytose [7]. C'est ce mécanisme qui est exploré ici.

La stabilité d'un morphogène dans l'espace extracellulaire dépend en premier lieu de ses propriétés biochimiques intrinsèques. Mais il est possible qu'il existe des mécanismes plus complexes chargés de la dégradation spécifique du morphogène, ou au contraire de sa modification pour le rendre plus stable.

Grâce au développement de techniques d'imagerie et à l'utilisation d'anticorps et de protéines de fusion morphogène-GFP (green fluorescent protein) ou morphogène-HRP (horse radish peroxidase), la distribution de ces morphogènes au cours du développement de la drosophile a été récemment décrite avec une très grande précision. Ces travaux, qui combinent des techniques de biologie cellulaire et de génétique, ont ainsi révélé que le mécanisme d'endocytose joue un rôle central dans la mise en forme des gradients de morphogène.

\section{Endocytose et transport des morphogènes}

L'hypothèse qu'un morphogène puisse se propager au sein d'un tissu dans des vésicules d'endocytose a été proposé dans le cas de Wingless. En effet, par immunolocalisation et analyse en microscopie électronique, Wingless est majoritairement détecté dans les endosomes des cellules épidermiques embryonnaires de la drosophile [8, 9]. Sur la base de cette observation, il a donc été suggéré que ce morphogène se propage au sein de ce tissu par transcytose « planaire » (c'est-à-dire dans le plan de l'épithélium), par analogie à la transcytose trans-épithéliale qui désigne le transport vésiculaire entre le domaine basal et le domaine apical des cellules épithéliales [10]. Ce modèle est renforcé par l'analyse d'embryons mutants pour le gène shibire, codant pour la dynamine, une GTPase requise pour l'endocytose dépendante de la clathrine. Dans des embryons déficients pour shibire, le champ d'action de Wingless est réduit [11]. Cependant, cette observation ne peut être généralisée à tous les tissus. Dans le disque d'aile de drosophile, par exemple, Strigini et al. [12] ont montré que l'endocytose n'est pas requise pour le transport de Wingless: Wingless se propage normalement dans des clones de cellules mutantes pour shibire. Ainsi, en fonction du tissu, un morphogène pourrait utiliser différents mécanismes pour atteindre les cellules les plus distantes.

À l'inverse de Wingless, Dpp semble, au moins en partie, se propager à travers les cellules du disque d'aile de drosophile par des cycles répétés d'endocytose et de re-sécrétion. Récemment, Entchev et al. [13] et Teleman et al. [14] ont analysé la formation du gradient de Dpp dans ce

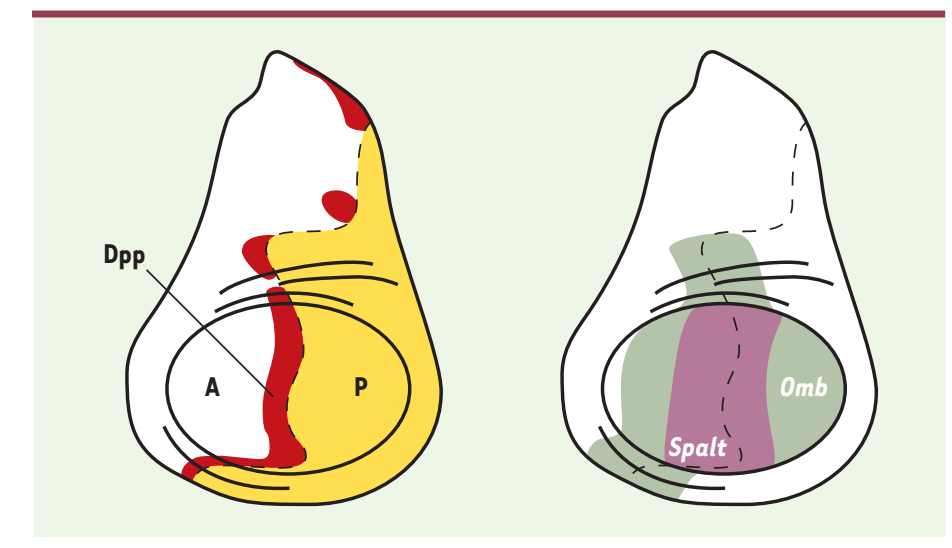

Figure 1. Dpp est un morphogène dans le disque imaginal d'aile. Le disque imaginal d'aile est constitué d'un compartiment antérieur ( $A$, blanc) et d'un compartiment postérieur ( $P$, jaune). Dpp est exprimé dans le compartiment antérieur dans une bande de cellules (en rouge). L'expression de deux gènes cibles de Dpp, spalt (en mauve) et optomotorblind (omb) (en vert), est indiquée sur le disque imaginal de droite. 
tissu, grâce à l'utilisation d'une protéine de fusion fluorescente, Dpp-GFP, biologiquement active. Lorsque DppGFP est produite dans le domaine d'expression de Dpp, c'est-à-dire à la frontière antéro-postérieure, elle se propage de part et d'autre de ce domaine en formant un gradient, et apparaît essentiellement dans des vésicules intracellulaires apicales. L'internalisation de Dpp-GFP dans ces vésicules est dépendante du récepteur de Dpp Thikvein (Tkv): dans des clones de cellules mutantes pour

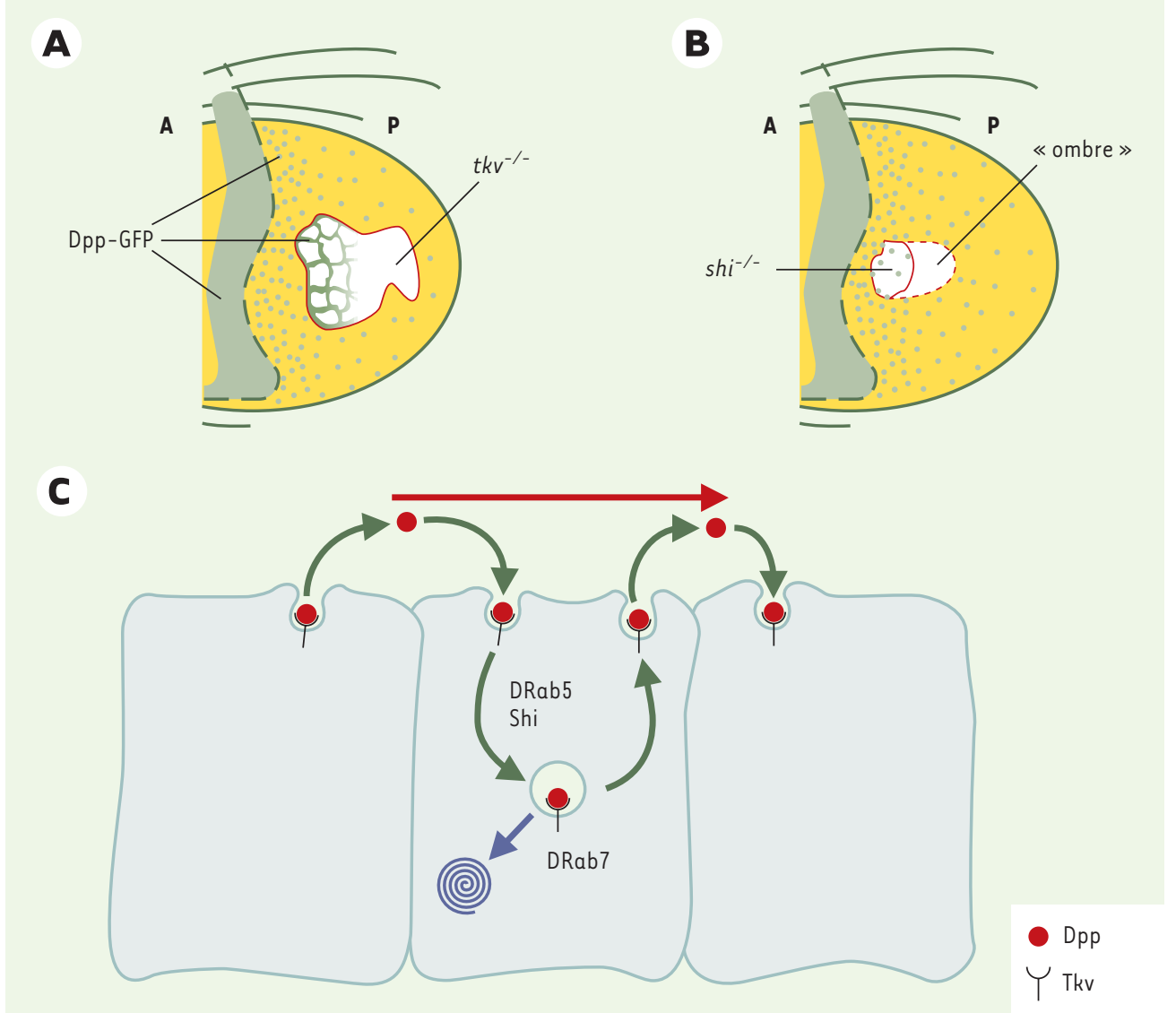

Figure 2. Dpp est transporté par transcytose. A. Dans un clone de cellules mutantes pour le récepteur de Dpp Thickvein (tkv) (clone $t k v^{-/-}$, délimité en rouge), la protéine de fusion Dpp-GFP (en vert) n'est plus transportée correctement; son mouvement est bloqué et elle s'accumule à la surface des cellules qui sont les plus proches de la source du morphogène. À l'extérieur du clone, Dpp-GFP est internalisé correctement par les cellules et se localise dans des vésicules intracellulaires (point vert). $\boldsymbol{B}$. Un clone de cellules homozygotes pour un allèle codant pour une isoforme thermosensensible de Shibire $\left(s h i^{-/-}\right)$est délimité par la ligne rouge. À température restrictive, la fonction de Shibire est bloquée dans les cellules du clone, qui sont alors incapables d'endocyter Dpp-GFP et donc de transmettre le morphogène aux cellules normales situées derrière le clone. Celui-ci se comporte comme une barrière, ce qui provoque l'apparition d'une ombre indiquée par la ligne pointillée rouge. Au sein du clone, quelques vésicules Dpp-GFP (en vert), formées peu avant le blocage de la fonction de Shibire, sont détectées. C. Modèle simplifié du processus de transcytose du morphogène Dpp dans le plan de l'épithélium du disque d'aile. Le mouvement net du morphogène est indiqué par la flèche rouge. Des vésicules transporteraient le morphogène d'un côté à l'autre de la cellule. Les protéines DRab5 et Shibire sont nécessaires à la formation de ces vésicules. La protéine DRab7 contrôle quant à elle le transfert de ces vésicules vers le compartiment de dégradation, les lysosomes. ce récepteur, la protéine fluorescente n'est plus présente dans des endosomes, mais s'accumule dans l'espace xtracellulaire autour des cellules les plus proches des ellules sources de Dpp, mais pas autour des cellules les plus distantes (Figure 2A). Ainsi, lorsque son récepteur est absent, Dpp-GFP n'est plus internalisé et semble immobilisé dans l'espace extracellulaire, suggérant que l'endocytose de la protéine via son récepteur est nécessaire à son mouvement [13]. Cependant, il n'est pas exclu qu'une partie du mouvement de Dpp ait lieu dans l'espace extracellulaire. En effet, si Dpp se déplaçait exclusivement dans des vésicules, il devrait s'arrêter à la première rangée de cellules mutantes dans ces clones. Or, Dpp peut encore s'accumuler sur quelques rangées de cellules n'exprimant pas son récepteur (Figure 2A).

Entchev et al. ont alors analysé le mouvement de Dpp-GFP dans des clones de cellules mutantes exprimant un allèle de shibire codant pour une protéine thermosensible. Après avoir produit des clones de cellules homozygotes pour cet allèle, puis induit une «vague » de propagation de Dpp-GFP via un pulse d'expression (pour les détails techniques, voir [13]), ils ont incubé les larves à température non permissive pour bloquer la fonction de Shibire. Ces clones de cellules mutantes induisent dans les cellules adjacentes situées derrière le clone une «ombre » dans le gradient du morphogène: en d'autres termes, le niveau de Dpp-GFP diminue dans les cellules situées derrière le clone, comme si les cellules mutantes incapables d'endocyter Dpp-GFP constituaient une barrière au mouvement de la protéine (Figure 2B). 
La protéine DRab5 est une petite GTPase requise pour la formation des endosomes (Figure 2C). Entchev et al. ont étudié l'effet de l'expression de l'isoforme sauvage ou d'une isoforme dominante négative de DRab5 sur la formation du gradient de Dpp [13]. L'expression de l'isoforme dominante négative a pour effet de bloquer l'endocytose dépendante de la clathrine. Lorsque cette isoforme est exprimée dans le compartiment postérieur, le domaine d'expression de spalt est restreint dans ce compartiment aux cellules adjacentes au domaine d'expression de Dpp, indiquant une réduction du champ d'action du morphogène. Inversement, l'expression de l'isoforme sauvage, qui augmente le taux d'endocytose, augmente aussi le domaine d'expression de spalt, indiquant qu'en condition normale le taux d'endocytose est limitant pour le transport de Dpp. L'ensemble de ces observations montre donc que l'endocytose de Dpp via son récepteur joue un rôle essentiel dans son transport, et suggère que sa propagation dans le disque d'aile s'effectue par un mécanisme de transcytose «planaire ».

Cependant, il convient de mentionner un article très récent de Lander et al. [15] qui suggère, à partir de modélisations mathématiques, qu'un défaut d'endocytose dans un groupe de cellules induirait une «ombre » derrière ce groupe même si le transport du morphogène s'effectue par libre diffusion, et non par transcytose. Dans le cas de la libre diffusion, les modèles mathématiques [16] prédisent qu'une grande quantité de récepteurs à la surface des cellules piégerait le morphogène et en ralentirait la diffusion; toutefois, la quantité de récepteur autorisant la libre diffusion ne semble pas suffisante pour assurer également la signalisation. Lander et al. proposent alors un modèle dans lequel l'endocytose du récepteur maintiendrait sa quantité effective à la surface à un taux suffisamment faible pour permettre la diffusion du morphogène. Afin que la signalisation soit malgré tout efficace, les complexes récepteur-ligand continueraient à signaler après leur internalisation. Dans ce modèle, le défaut d'endocytose provoquerait la formation d'une ombre non pas forcément parce que le morphogène est transporté par transcytose, mais en raison d'une accumulation du récepteur à la surface qui ralentirait la libre diffusion du ligand. Bien que cette étude ne prouve pas que les morphogènes diffusent librement, elle conduit à réexaminer les arguments en faveur de la transcytose planaire et démontre la valeur indiscutable de la modélisation mathématique pour la compréhension des processus biologiques.

\section{Endocytose et dégradation des morphogènes}

L'analyse de la propagation de Dpp-GFP montre que cette protéine se déplace rapidement dans le disque d'aile. En dépit de cette vitesse de propagation élevée, un gradient de concentration se forme et reste stable au cours $\mathrm{du}$ temps: en d'autres termes, Dpp-GFP ne finit pas par « remplir » tout le disque au bout d'un certain temps. Sur simulation informatique, il suffit d'introduire un faible pourcentage de dégradation de la protéine pour qu'un gradient stable se forme. Ainsi, il est probable qu'une fraction de Dpp-GFP soit dégradée dans le disque d'aile, afin de stabiliser le gradient. Une façon de dégrader la protéine serait de détourner une partie des endosomes qui la contiennent vers le compartiment endocytique de dégradation, les lysosomes. Pour tester cette hypothèse, Entchev et al. ont engendré une isoforme constitutivement activée de DRab7, une petite GTPase contrôlant le transfert des endosomes vers les lysosomes (Figure 2C). L'expression de cette isoforme constitutivement active de DRab7 a pour effet d'augmenter la fraction des endosomes détournée vers les lysosomes, et donc d'augmenter le taux de dégradation. La surexpression de cette isoforme dans le compartiment postérieur du disque d'aile a pour effet de réduire, dans ce compartiment, le domaine d'expression de spalt aux cellules adjacentes à celles exprimant Dpp, ce qui indique une réduction du champ d'action de ce morphogène. Sur la base de ces résultats, Entchev et al. suggèrent que le détournement des endosomes contenant Dpp vers les lysosomes limite son champ d'action et stabilise son gradient de concentration. La forme (ou pente) du gradient de Dpp dépendrait alors de l'équilibre entre recyclage vers la membrane et dégradation dans les lysosomes [13].

Récemment, nous avons démontré que le routage des endosomes vers les lysosomes contrôle également la distribution du morphogène Wingless dans l'épiderme de l'embryon de drosophile [17]. Au cours de l'embryogenèse, Wingless est exprimé dans une seule rangée de cellules au sein de chaque segment (Figure 3). II induit la production de cuticule dite «nue » par les cellules qui reçoivent son signal. Les cellules qui ne reçoivent pas son signal vont en revanche produire de la cuticule avec des denticules (Figure 3). II a été observé que Wingless agit sur une distance plus grande du coté antérieur que du coté postérieur, du fait de sa distribution asymétrique; pourtant, Wingless est initialement distribué symétriquement de part et d'autre de son domaine d'expression (Figure 3) [18]. Grâce à l'utilisation d'une protéine de fusion HRP-Wingless, nous avons montré que la distribution asymétrique de Wingless est due à la dégradation préférentielle du morphogène dans les lysosomes des cellules postérieures à sa source [17].

Pour étudier le devenir d'un ligand dans le compartiment endocytique, l'utilisation d'une protéine de fusion HRP présente plusieurs avantages [19]. Tout d'abord, I'acti- 
vité enzymatique de cette protéine est facilement détectable dans les tissus qui l'expriment. II suffit pour cela d'incuber les tissus dans une solution contenant le substrat de l'enzyme HRP, le DAB (diaminobenzydine); après réaction avec l'enzyme, le substrat forme un précipité brun, dense aux électrons, détectable en microscopie photonique et électronique. La propriété du précipité d'être détectable en microscopie électronique permet l'analyse détaillée de la localisation subcellulaire de la protéine de fusion, et l'identification du type d'endosome qui la contient (endosome précoce, lysosome...). Le deuxième avantage de la protéine HRP est qu'elle est stable dans tout le compartiment endocytique, y compris dans les lysosomes. Ainsi, si un ligand est destiné à être dégradé dans les lysosomes après endocytose, une protéine chimère entre ce ligand et la protéine HRP révélera ce destin: on observera une accumulation de la moitié HRP de la chimère dans les lysosomes. Lorsque I'on exprime HRP-Wingless dans l'embryon de drosophile, on observe une accumulation massive de la partie HRP dans les lysosomes des cellules postérieures à sa source, alors que dans les cellules antérieures, la protéine chimère
A

A

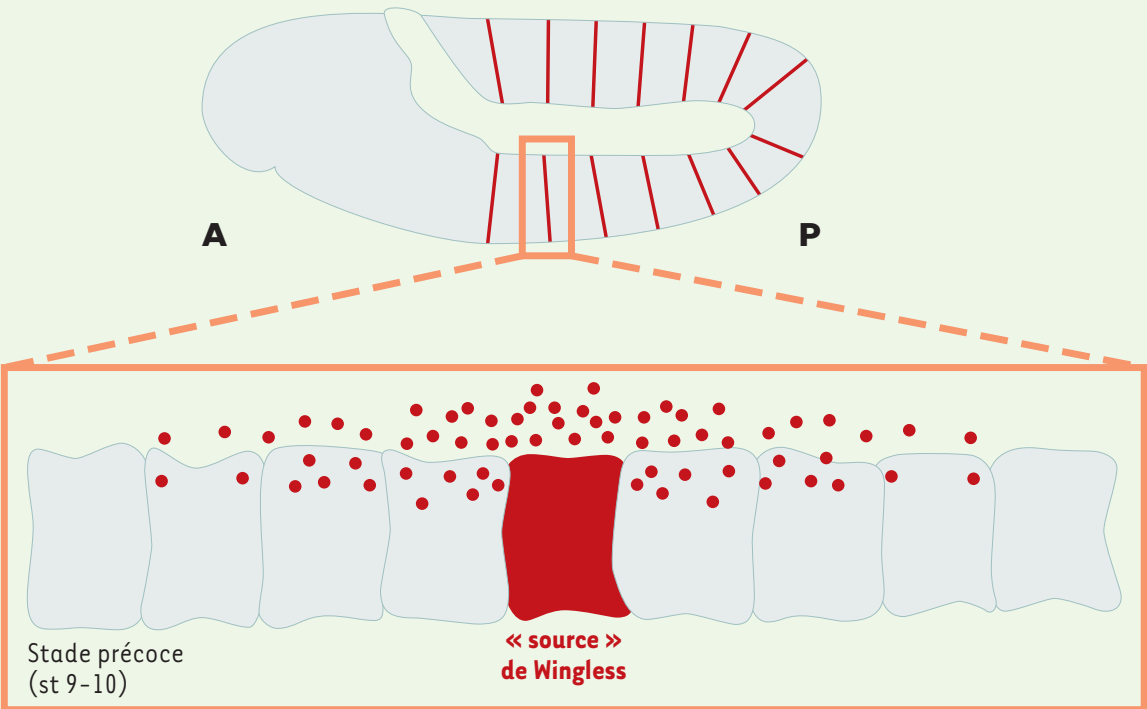

c
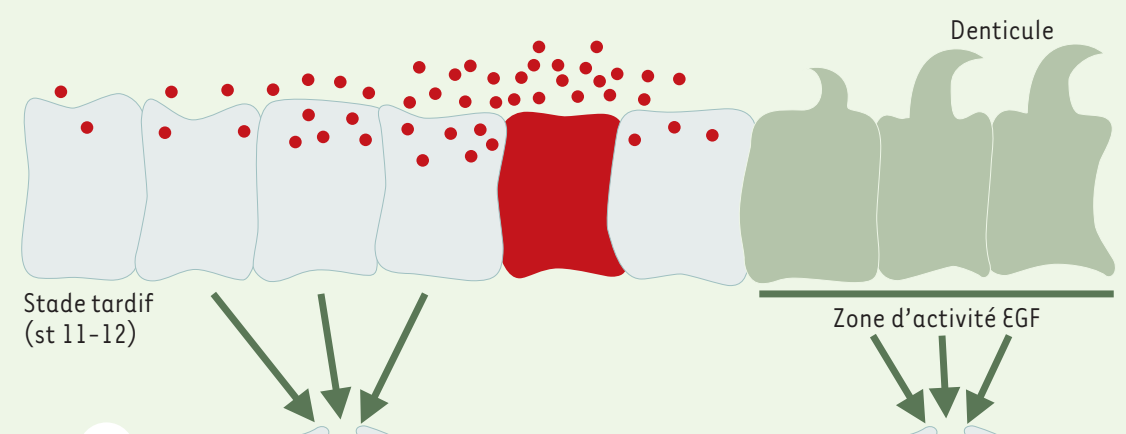

D

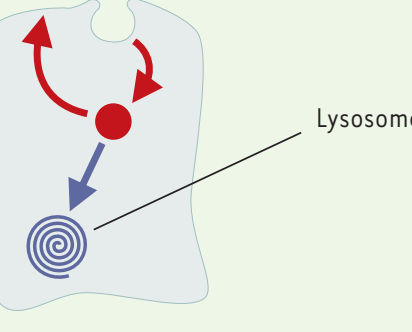

Figure 3. La distribution de Wingless est contrôlée par sa dégradation dans les lysosomes. A. Rangée de cellules (bordure rouge) exprimant Wingless dans chaque segment d'un embryon au stade (st) $10-11$ ( $6 \mathrm{~h}$ après la ponte à $\left.25^{\circ} \mathrm{C}\right)$; A: compartiment antérieur, $P$ : compartiment postérieur. $B$ et $C$. Coupe de cellules de l'épiderme au niveau d'un seul segment, à deux stades différents («précoce », stade 9-10, et «tardif », stade 1112), séparés de $2 \mathrm{~h}$ à $25^{\circ} \mathrm{C}$. La cellule rouge est dans les deux cas celle qui exprime Wingless. Au stade précoce (B), la distribution de Wingless est symétrique, de part et d'autre de son domaine d'expression. Au stade tardif (C), la distribution de Wingless devient asymétrique: du côté postérieur, Wingless disparaît des cellules qui reçoivent le signal EGF (en vert) et qui formeront des denticules. D. Au stade tardif: du coté antérieur (à gauche), Wingless est stable, possède une activité de signal effective et peut être détecté, car il n'est pas (ou très peu, flèche bleue) détourné vers les lysosomes après endocytose; il

pourrait éventuellement être redirigé vers la membrane (flèche rouge). Du côté postérieur, Wingless disparaît rapidement, n'est plus détectable et ne signale pas, car il est rapidement détourné après endocytose vers les lysosomes pour y être dégradé (flèche rouge), et n’est vraisemblablement pas (ou très peu) recyclé vers la membrane (flèche bleue). 
reste (plus longtemps) intacte: une faible activité HRP est détectée dans les lysosomes. Cette observation montre la dégradation préférentielle de Wingless dans les cellules postérieures, ce phénomène pouvant être responsable de la distribution asymétrique de la protéine (Figure 3). Pour tester cette hypothèse, nous avons étudié la distribution de Wingless lorsque l'activité de deux gènes, clathrine et deep-orange, requis respectivement pour l'endocytose et la genèse des lysosomes, est réduite $[20,21]$. Dans les deux cas, on observe une accumulation de Wingless dans les cellules postérieures, qui s'accompagne d'un excès de signal: les cellules postérieures, qui forment normalement des denticules, produisent alors de la cuticule nue. Ces résultats montrent que l'endocytose et la dégradation dans les lysosomes sont importantes pour contrôler la distribution et l'activité de Wingless dans l'embryon de drosophile. II faut noter que le taux de dégradation est réglé à la fois spatialement et temporellement, et que cette régulation est sous le contrôle d'une autre voie de signalisation, la voie de l'EGF (epidermal growth factor) (Figure 3).

Si ces résultats montrent que l'endocytose est requise pour la dégradation de Wingless dans l'embryon, ils suggèrent aussi qu'elle n'est pas requise pour son transport, au moins dans les cellules postérieures: en effet, lorsque l'activité du gène clathrine, requis pour l'endocytose, est diminuée, Wingless peut se propager et agir du côté postérieur. Cela n'exclut toutefois pas que du côté antérieur (où Wingless est normalement actif), l'endocytose et le recyclage participent au moins en partie à sa propagation, mais cette hypothèse reste encore à tester.

\section{Conclusions}

L'endocytose est souvent perçue comme une fonction « de ménage », dont les mécanismes sont identiques de la levure à l'homme. Ces études réalisées chez la drosophile montrent au contraire que le contrôle spatial et temporel de l'endocytose et du trafic intracellulaire de molécules spécifiques est fondamental dans un processus biologique tel que la formation d'un gradient de morphogène. Cette spécificité d'action a encore été démontrée récemment par l'identification du gène correspondant à la mutation open brain chez la souris, qui induit une hyperactivation de la voie Hedghehog. Ce gène code pour Rab23, une petite GTPase contrôlant le trafic intracellulaire des endosomes [22]. Ainsi, chez les vertébrés, la signalisation Hedgehog pourrait également être contrôlée par endocytose et routage intracellulaire. Cependant, même si l'implication de ces processus lors de la formation des gradients de morphogène est aujourd'hui très vraisemblable, la façon dont il sont contrôlés reste obs- cure. Par exemple, la direction du mouvement des vésicules transportant Dpp après endocytose est-elle contrôlée? En d'autres termes, les vésicules sont-elles dirigées vers un endroit particulier de la membrane de la cellule pour la re-sécrétion, de façon à augmenter (ou réduire) la vitesse de propagation de Dpp? Dans le cas de Wingless, quel est le récepteur (ou complexe de récepteurs) responsable de son internalisation? Quelles sont les molécules contrôlant le détournement des endosomes vers les lysosomes dans les cellules postérieures? Comment ces molécules sont-elles contrôlées par la voie EGF, qui accélère la dégradation de Wingless dans certaines cellules de l'épiderme embryonnaire de drosophile? La synthèse qui s'opère actuellement entre biologie du développement, génétique et biologie cellulaire, ainsi que l'utilisation de la drosophile comme système modèle devraient permettre d'apporter dans les années à venir des réponses à toutes ces questions. $\diamond$

\section{REMERCIEMENTS}

Je remercie K. Rizzoti, A. Vincent et J.P. Vincent pour leurs commentaires sur ce manuscrit.

\section{SUMMARY}

Morphogens and endocytosis

During development, secreted signaling proteins of the Wingless/Wnt, Hedgehog and Decapentaplegic (Dpp)/ Bone Morphogenic Protein (BMP) families act as morphogens. Previous work had shown that these molecules act directly on distant cells, although until recently nothing was known about how they reach those distant cells. During the past two years, work carried out on Drosophila using genetic and cell biology approaches have revealed that endocytosis plays a central part in the mechanisms that control the spread of morphogens. $\Delta$

\section{RÉFÉRENCES}

1. Wolpert L. One hundred years of positional information. Trends Genet $1996 ; 12$ : 359-64.

2. Nellen D, Burke R, Struhl G, Basler K. Direct and longrange action of a DPP morphogen gradient. Cell 1996; 85: 357-68.

3. Lecuit T, Brook WJ, Ng M, Calleja M, Sun H, Cohen SM. Two distinct mechanisms for long-range patterning by Decapentaplegic in the Drosophila wing. Nature 1996; 381: 387-93. 
4. Reichsman F, Smith L, Cumberledge $\mathrm{S}$. Glycosaminoglycans can modulate extracellular localization of the Wingless protein and promote signal transduction. J Cell Biol 1996; 135: 819-27.

5. Bradley RS, Brown AM. The proto-oncogene int-1 encodes a secreted protein associated with the extracellular matrix. EMBO 1990; 9: 1569-75.

6. Cumberledge $S$, Reichsman F. Glycosaminoglycans and WNT's: just a spoonful of sugar helps the signal go down. Trends Genet 1997; 13: 421-3.

7. Pfeiffer S, Vincent JP. Signalling at a distance: transport of Wingless in the embryonic epidermis of Drosophila. Semin Cell Dev Biol 1999; 10: 303-9.

8. Van den Heuvel M, Nusse R, Johnston P, Lawrence P. Distribution of the wingless gene product in Drosophila embryos: a protein involved in cell-cell communication.
Cell 1989; 59: 739-49.

9. Gonzalez F, Swales L, Bejsovec A, Skaer H, Martinez Arias A. Secretion and movement of wingless protein in the epidermis of the Drosophila embryo. Mech Dev 1991; 35: 43-5.

10. Mostov KE, Verges M, Altschuler $Y$. Membrane traffic in polarized epithelial cells. Curr Opin Cell Biol 2000; 12: 483-90.

11. Moline MM, Southern C, Bejsovec A. Directionality of wingless protein transport influences epidermal patterning in the Drosophila embryo. Development 1999 ; 126: 4375-84.

12. Strigini M, Cohen SM. Wingless gradient formation in the Drosophila wing. Curr Biol 2000; 10: 293-300.

13. Entchev EV, Schwabedissen A, Gonzalez-Gaitan M. Gradient formation of the TGF-beta homolog Dpp. Cell 2000; 103: 981-91.

14. Teleman AA, Cohen SM. Dpp gradient formation in the Drosophila wing imaginal disc. Cell 2000; 103: 971-80.

15. Lander AD, Nie Q, Wan YF. Do morphogen gradient arise by diffusion? Dev Cell 2002; 2: 785-96.

16. Kersberg M, Wolpert $\mathrm{L}$. Mechanisms for positional signalling by morphogen transport: a theoretical study. J Theor Biol 1998; 191: 103-14.

17. Dubois $L$, Lecourtois $M$, Alexandre C, Hirst $\varepsilon$, Vincent JP. Regulated endocytic routing modulates wingless signaling in Drosophila embryos. Cell 2001; 105 : 613-24.

18. Sanson B, Alexandre C, Fascetti N, Vincent JP. Engrailed and hedgehog make the range of Wingless asymmetric in Drosophila embryos. Cell 1999; 98 : 207-16.

19. Sunio A, Metcalf AB, Kramer $H$. Genetic dissection of endocytic trafficking in Drosophila using a horseradish peroxidasebride of sevenless chimera: hook is required for normal maturation of multivesicular endosomes. Mol Biol Cell 1999; 10: 847-59.

20. Bazinet $C$, Katzen $A L$, Morgan M, Mahowald AP, Lemmon SK. The Drosophila clathrin heavy chain gene: clathrin function is essential in a multicellular organism. Genetics 1993; 134: 1119-34.

21. Sevrioukov $\varepsilon A, H e J P$, Moghrabi N, Sunio A, Kramer $H$. A role for the deep orange and carnation eye color genes in lysosomal delivery in Drosophila. Mol Cell 1999; 4: 479-86.

22. Eggenschwiler JT, Espinoza $\varepsilon$, Anderson KV. Rab23 is an essential negative regulator of the mouse Sonic hedgehog signalling pathway. Nature 2001; 412 : 194-8.

TIRÉS À PART

L. Dubois

PUB FLAMMARION FILMS CHEZ CORLET 